\title{
South African Sports Medicine Association (SASMA) consensus statement on policy for managing athletes with disorders of sexual development (DSD)
}

SASMA views disorders of sexual development (DSD) as a medical condition that has profound physical and psychological effects on not only the individuals affected, but also their families. Like any other disorder, this condition has to be managed with a view to offer the best outcomes for affected individuals. When the attending physician identifies stigmata suggesting DSD in a newborn there should be proper consultation and education of parents.

Challenges in managing DSD include the following:

1. Many of these children are born at home in rural areas and subsequently may not access formal medical structures where the DSD can be detected and managed.

2. In this situation where the DSD is not detected the parent/s will decide on the gender; they usually assign a female gender.

3. Issues of sexuality are often not discussed within families until puberty, which is when the different traits start showing.

4. There is limited knowledge of DSD in the population, and some individuals will only be diagnosed in adulthood when they encounter problems with sexuality or infertility. In a sporting environment however, these individuals will stand out earlier, particularly if they were raised as girls and develop masculine features.

The South African Sports Medicine Association re-iterates the following ethical considerations for practitioners dealing with individuals with DSD:

- Adherence to strict confidentiality in any medical consultations

- The necessity to obtain fully informed, written consent for any investigations

- Cross-referral within a specialised team consisting of

- gynaecologist/s

- endocrinologist/s

- urologist/s

- psychologist/s

- physician/s

- Complete disclosure of information and education of the affected individual regarding the implications of both performing the tests and the findings thereof.
Further participation in sports will be determined by the diagnosis reached, management of the condition with informed consent from the individuals, and guidelines given by the sporting bodies.

Strategies should be implemented to address DSD in athletes before they achieve a high profile and compete internationally. This approach may help avoid humiliation and exposure of their intimate details to the public. The following strategy is recommended:

- Education rollout at schools, sporting bodies, clinics and the public via media and/or formal education.

- Nursing staff at clinics to be educated to observe and advise parents, and refer for specialised opinion.

- Coaches and teachers, who are usually the first contact with talented athletic children, to be educated on the sensitivity of this problem, and how to refer for proper management.

- All athletes who start competitive international sports from U17 IAAF level meets should have a medical and sexual health screening by a physician (preferably of the same gender). This should not be an invasive examination, but the physician should know the stigmata to look for. Any suspected cases of DSD can then be identified and examined more thoroughly.

- Fully informed consent from athlete and parents/guardians in the under-age athlete to be obtained.

- Extensive psychological support to be given to the athlete and his/her family.

A concern with this approach might be that athletes who already suspect that they are different, may withdraw from competition to avoid the examination. In order to pre-empt and overcome this, prior education on the rules of sporting bodies and an emphasis on confidentiality of all medical findings is important. In particular it should be emphasised that the aim of the medical strategy is to identify and manage conditions so that the athlete can continue with sport.

SASMA, as the national umbrella body for sports medicine, commits itself within the bounds of medical ethics and sound clinical practice, to co-operate with other national representative sports bodies for the benefit of the health of South African athletes.

\section{SASMA Executive Committee}

August 2010 